\title{
Comparison of Interpretive Description and Qualitative Description in the Nursing Scope
}

\author{
Azam Ghorbani' \\ ORCID: 0000-0003-1240-745X \\ Pegah Matourypour' \\ ORCID: 0000-0002-9476-0267
}

'Tehran University of Medical Science. Teerã, Irã.

How to cite this article:

Ghorbani A, Matourypourl P. Comparison of Interpretive Description and Qualitative Description in the Nursing

Scope. Rev Bras Enferm. 2020;73(1):e20190339. doi: http://dx.doi.org/10.1590/0034-7167-2019-0339

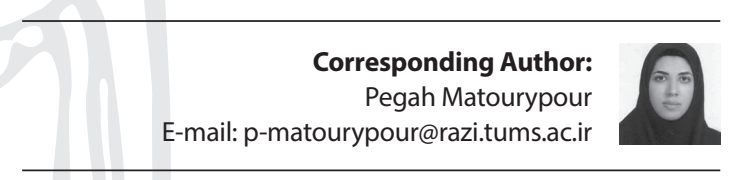

EDITOR IN CHIEF: Dulce Aparecida Barbosa
Nowadays, researchers are looking for methods according to the epistemological basis and systematic reasoning and that lead to knowledge broadening for the field. Thus, researchers began changing rules and methodological principles. Interpretive Description (ID) is a qualitative research method that assesses the clinical phenomena related to the field. Themes and patterns are extracted from mental perceptions and create a description that lead to clinical understanding ${ }^{(1)}$. This method was presented by Sally Thorne et al. in 1997, and is one of the inductive analysis methods helping with the understanding of clinical phenomena. In fact, it is a method moving beyond the dominant approaches in nursing research, being an evolution in the qualitative research method in the nursing field ${ }^{(2)}$.

\section{Interpretive Description Basis}

ID is a small scale qualitative research about a phenomenon regarded in the field that aims to extract themes and patterns. This approach begins with the critical analysis of clinical and theoretical knowledge in the field. It develops the initial conceptual framework, however it does not enter procedures details. Instead, this approach explains the overall criteria used for assessing design decisions and adapting them to qualitative methods ${ }^{(1)}$. This method has an adjusted methodological framework, and its primary goal is to create a clinical understanding, while its secondary goal is to use this clinical understanding in patient care $^{(3)}$.

Multiple data collection methods are used in this method. The producedinterpretive report is based on informed questions, thinking and reflection techniques use, and critical thinking. Sampling is based on purpose and is often theoretical. Moreover, participants are aware of the studied phenomenon. Different validation methods including concurrent collection and analysis, continuous comparative analysis, and repeated analysis are used. The ID result is a conceptual coherent description describing the studied phenomenon using thematic patterns ${ }^{(2)}$.

In nursing, several questions are about patient experience: how is it like having a specific disease? What thoughts and feelings individuals experience while faced with specific challenges? How do they understand their need for receiving care? Data collection and sampling methods are extracted from research questions. Analytical process in an ID includes testing and challenging the initial interpretations and conceptualizing a coherent final result. Finally, the studied phenomenon is presented in a significant new method instead of providing abstract interpretations from the initial descriptive claims ${ }^{(2)}$.

\section{Comparison of Interpretive Description and Qualitative Description}

Knowledge about qualitative description (QD) and its use in the health research field is limited and often criticized for its simplicity and lack of accuracy. However, QD is appropriate in mixed methods to obtain first-hand knowledge of patients, relatives, or professional experiences. In addition, resource and time constraint are advantages of this method. QD is different from several other methods in terms of quality. The objective of this method is not a deep description such as ethnography, nor theorization like the Grounded Theory, 
rather an explanation of a phenomenon such as phenomenology. Instead, this method aims at direct and rich description of an experience or event. Unlike other qualitative research methods where conceptualization or data analysis are in relation to the current theories, the final result is a description of informed samples experiences with a similar language to the samples. In the analysis process, the researcher works more on the data and does not move away from them. Targeted sampling with maximum diversity is suggested. Semi-structured interviews with open-ended questions are used. The interviews may be individual or focus group, being more appropriate for obtaining a broader insight into the subject. The researcher raises the questions like who, how, where, and why about the desired experience. Special events are observed and the documents or other relevant items are reviewed. In data analysis, qualitative content analysis (QCA) is used with a modified coding system, which is appropriate for data collection. The analysis aims to understand the latent variables (which are useful for explaining the concept and tooling). The result is direct data description in such a way to be proportional to the data ${ }^{(4)}$. One of the strengths of this method is that it can provide useful information before developing a questionnaire or an intervention, and gives us an initial insight into the specific issue. Clinical individuals use qualitative research for small research projects while QD is an appropriate method for obtaining the perspective of patients and their families. QD should not be confused with ID, because the goal of ID, in addition to mere description, is a deep conceptual description and understanding of the phenomenon, while QD does not go far from the data. Analytical such as theorization, recontextualization, and composition are more highlighted in ID ${ }^{(5)}$.

\section{Relationship Between Interpretive Description and The Grounded Theory}

ID is influenced by the Grounded Theory, phenomenology, ethnography, naturalistic research in design presentation, sample, data collection, and analysis. The Grounded Theory effect on this method is completely obvious. Indeed, the Grounded Theory makes up the ID epistemological basis. Some researchers believe that ID is not a new approach of qualitative research, but is part of the Grounded Theory in numerous ways. The question arising here is why should a method be invented being so similar to the other method? It should be said that in ID, the researcher prefers to focus on individuals rather than on concepts, being developed by using various methods (such as Charmaz). In order to answer the question, "why do we not use a qualitative content analysis or interpretive phenomenology?", it can be stated that it is due to description and interpretation that exist in this method ${ }^{(1)}$. In ID, researchers often provide a coherent conceptual description, having thematic patterns and identifying the studied phenomenon in addition to reporting the inevitable personal differences.

\section{CONCLUSION}

An ID aimed at assessing the clinical phenomena provides an opportunity to avoid the limiting principles of traditional research methods for researchers. Analysis in this method is based on inductive reasoning in a valid and acceptable manner. The process completely depends on the researcher and requires data, imagination, and conceptual creativity immersion. Indeed, ID sues a conceptual and coherent description to better understand the clinical phenomena and provide findings for clinical use. QD is a new method in the qualitative research field, being used in questionnaire design and combined research. It is a direct description of the phenomenon and can be used where there is no deep data analysis. Despite the similarities between QD and ID, they are two different methods. ID is a more conceptual description of the phenomenon and provides more data analysis than QD, while QD does not go far from the original data. The emergence of new methods in the nursing research methods field is a step towards application of research in this field.

\section{REFERENCES}

1. Bertero C. Developing qualitative methods or "same old wine in a new bottle. Int J Qualit Stud Health Well-being. 2015;10:27679. doi: 10.3402/qhw.v10

2. Thorne S, Reimer Kirkham S, O'Flynn-Magee K. The Analytic Challenge in Interpretive Description. Int J Qualit Meth [Internet]. 2004[cited 2019 Apr 02]3(1). Available from: https://sites.ualberta.ca/ iiqm/backissues/3_1/pdf/thorneetal.pdf

3. Stubbs MJ. Qualitative description of the adult patient experience of cancer-related cachexia (CRC) : a pilot study Palmerston North, New Zealand: Massey University; 2008

4. Sandelowski M. Focus on research methods. whatever happened to qualitative description? Res Nurs Health. 2000;23(4):334-40. doi: 10.1002/1098-240X(200008)23:43.0.CO;2-G

5. Asbjoern Neergaard M, Olesen F, Sand Andersen R, Sondergaard J. Qualitative description: the poor cousin of health research? BMC Med Res Methodol [Internet]. 2009[cited 2019 Apr 02];9:52. Available from: https://bmcmedresmethodol.biomedcentral.com/ articles/10.1186/1471-2288-9-52 